OPEN ACCESS

Edited by:

Guy Smagghe,

Ghent University, Belgium

Reviewed by:

Takashi Koyama,

University of Copenhagen, Denmark

Christen Kerry Mirth,

Monash University, Australia

*Correspondence:

Yi-Fan Zhai

zyifan@saas.ac.cn

Specialty section:

This article was submitted to

Invertebrate Physiology,

a section of the journal

Frontiers in Physiology

Received: 10 April 2018 Accepted: 02 November 2018 Published: 20 November 2018

Citation

Yin Z-J, Dong $X$-L, Kang $K$, Chen H, Dai X-Y, Wu G-A, Zheng L, Yu $Y$ and Zhai Y-F (2018) FoxO

Transcription Factor Regulate Hormone Mediated Signaling on

Nymphal Diapause.

Front. Physiol. 9:1654.

doi: 10.3389/fphys.2018.01654

\section{FoxO Transcription Factor Regulate Hormone Mediated Signaling on Nymphal Diapause}

\author{
Zhen-Juan Yin', Xiao-Lin Dong'2, Kui Kang ${ }^{3}$, Hao Chen', Xiao-Yan Dai', Guang-An Wu',2, \\ Li Zheng ${ }^{1}$, Yi Yu ${ }^{1}$ and Yi-Fan Zhai ${ }^{1,2,4 *}$
}

${ }^{1}$ Institute of Plant Protection, Shandong Academy of Agricultural Sciences, Jinan, China, ${ }^{2}$ College of Agriculture, Yangtze University, Jingzhou, China, ${ }^{3}$ School of Life Sciences, Sun Yat-sen University, Guangzhou, China, ${ }^{4}$ College of Life Sciences, Shandong Normal University, Jinan, China

Diapause is a complex physiological adaptation phenotype, and the transcription factor Forkhead-box $\mathrm{O}(\mathrm{FoxO})$ is a prime candidate for activating many of its diverse regulatory signaling pathways. Hormone signaling regulates nymphal diapause in Laodelphax striatellus. Here, the function of the FoxO gene isolated from L. striatellus was investigated. After knocking-down $\angle s F O x O$ in diapausal nymphs using RNA interference, the titers of juvenile hormone III and some cold-tolerance substances decreased significantly, and the duration of the nymphal developmental period was severely shorted to 25.5 days at $20^{\circ} \mathrm{C}$ under short day-length (10 L:14 D). To determine how LsFoxO affects nymphal diapause, analyses of RNA-sequencing transcriptome data after treatment with LsFoxO-RNA interference was performed. The differentially expressed genes affected carbohydrate, amino acid and fatty acid metabolism, and phosphatidylinositol 3-kinase/protein kinase B signaling pathway. Thus, LsFoxO acts on L. striatellus nymphal diapause and is, therefore, a potential target gene for pest control. This study may lead to new information on the regulation of nymphal diapause in this important pest.

\section{Keywords: FoxO, RNAi, nymphal diapause, Laodelphax striatellus, RNA-sequencing (RNA-Seq)}

\section{INTRODUCTION}

Insects have evolved multiple strategies to adapt to environmental changes, such as diapause and migration. Diapause enables insects to decrease metabolism, arrest development and increase stress resistance under unfavorable conditions, and it is regulated by external environmental signals and internal genetic factors (Denlinger, 2002, 2008). Most insects rely on photoperiod and temperature signals to reach diapause, and there are several regulatory features of diapause, such as hormonal molecular regulation, the circadian clock and energy utilization (Eizaguirre et al., 1998; Anspaugh and Roe, 2005; Xu et al., 2012; Kauranen et al., 2016; Li et al., 2017). In addition, some cold tolerance substances accumulate and key enzymes catalyze, such as trehalase (TRE), sorbitol dehydrogenase (SDH), and pyruvate kinase (PK), etc., to improve the cold tolerance and overcome severe winter environments, (Rozsypal et al., 2013; Zhai et al., 2016). In addition, diapause typically occurs at a 
specific developmental stage for each species, such as the embryo, larvae/nymph, pupae or adult (Kostal, 2006; Williams et al., 2006; Zhang et al., 2013; Fan et al., 2017). Larval/nymphal diapause occurs because many insect species overwinter as larvae/nymph, in which the molting processes is usually arrested, and some related signaling pathways are suppressed, such as hormone and energy metabolism (Hahn and Denlinger, 2011; Jindra et al., 2013).

The Forkhead box (Fox) proteins form a family of transcription factors that has several subclasses. The member proteins are diverse but are characterized by a conserved DNA-binding domain. FoxO is the main transcriptional effector of the insulin signaling pathway and is normally suppressed in the presence of insulin (Martins et al., 2016). The insulin signaling pathway plays a critical role in regulating diapause in some invertebrates, such as Caenorhabditis elegans, Drosophila melanogaster, and Culex pipiens (Junger et al., 2003; Sim and Denlinger, 2008; Matsunaga et al., 2018). High insulin levels activate the phosphatidylinositol 3-kinase/protein kinase $\mathrm{B}$ (PI3K/Akt) pathway, which in turn phosphorylates FoxO, promoting FoxO inactivation. In $C$. pipiens, the shutdown of insulin signaling prompts the activation of downstream FoxO and leads to the adult diapause phenotype (Sim et al., 2015).

The small brown planthopper, Laodelphax striatellus Fallén (Hemiptera: Delphacidae), is a notorious pest in a variety of graminaceous crop systems, including rice, wheat, corn and barley (Kisimoto, 1989). It causes serious damage to plants due to the transmission of viruses associated with plant diseases, such as rice stripe virus and rice black-streaked dwarf virus (Otuka, 2013). L. striatellus exhibits more cold resistance than other rice planthopper species, Nilaparvata lugens (Stål) and Sogatella furcifera (Horváth), and the most L. striatellus northern populations showed the highest diapause incidence and a longer critical photoperiod (Hou et al., 2016). L. striatellus nymphal diapause has been studied under different laboratory conditions; however, little research has focused on the molecular regulatory mechanisms related to nymphal diapause (Kisimoto, 1989; Wang et al., 2014). In our previous study, we determined the 4thinstar nymph as the main diapause stage through investigation under field and laboratory conditions (Zhai et al., 2018). Here, we characterized the functions of the LsFoxO gene in nymphal diapause. The injection of $d s L s F o x O$ significantly altered the levels of some cold-tolerance substances, metabolic enzymes activities and hormone titers, and the duration of diapause in nymphs was shortened.

\section{EXPERIMENTAL SECTION}

\section{Ethics Statement}

The small brown planthopper, Laodelphax striatellus is an economically important pest insect in East Asia, which attacks a wide range of graminaceous crops. The field studies did not involve endangered or protected species, and no specific permissions were required for our research activities in these locations.

\section{Insect Rearing}

We obtained the original L. striatellus colony from the Shandong Rice Research Institute (SRRI; Shandong, China) in 2010. These insects were reared on fresh rice seedlings and maintained in the laboratory at $25 \pm 1^{\circ} \mathrm{C}$ under a $16 \mathrm{~L}: 8 \mathrm{D}$ photo regime and $70-80 \%$ relative humidity. Newly hatched 1 st instar nymphs were reared on fresh rice seedlings at $20^{\circ} \mathrm{C}$ under long day-length (16 L: $8 \mathrm{D})$, which resulted in all 4th instar nymphs individuals continuing through direct development (non-diapause). On the contrary, newly hatched 1st instar nymphs were reared at $20^{\circ} \mathrm{C}$ under short day-length (10 L:14 D), resulting in substantially all 4th instar nymphs individuals entering nymphal diapause, and developmental delay often characterized nymph population diapause (Wang et al., 2014; Zhai et al., 2018).

\section{The Cloning and Sequence Analyses of LsFoxO}

The Total RNA Kit II (Omega Bio-Tek, Norcross, GA, United States) was used to isolate the total RNA from larvae and pupae of L. striatellus, and first strand cDNA synthesis was performed using the 1st Strand cDNA Synthesis Kit (TaKaRa, Tokyo, Japan). Two pairs of intermediate fragments primers were designed from the L. striatellus transcriptome database by local blast (Table 1). The full-length complementary DNA (cDNA) of LsFoxO was cloned using the rapid amplification of cDNA ends RACE kit (Takara, Japan), and the evolutionary analyses were conducted using MEGA software, version 4.0 .

TABLE 1 | Primers used in this study.

\begin{tabular}{|c|c|}
\hline Primer name & Primer sequences $\left(5^{\prime}-3^{\prime}\right)$ \\
\hline \multicolumn{2}{|c|}{ Intermediate fragments primers } \\
\hline LsFoxO-F1 & GATAGGTGAGATGTCCGAGTGA \\
\hline LsFoxO-F2 & GCAGTAGTTGGTGTTGGTTGT \\
\hline LsFoxO-R1 & GCTGCTGCGTGAAGTTGAA \\
\hline LsFoxO-R2 & TGATGAGGTCGGCGTAGGA \\
\hline \multicolumn{2}{|c|}{ For cDNA cloning } \\
\hline $5^{\prime}-\angle S F O X O-1$ & TGTGTGATGAGGTCGGCGTAGGAGA \\
\hline $5^{\prime}-$ LsFoxO-2 & CAAATCCTCACTCGGACATCTCAC \\
\hline $3^{\prime}-$ LsFoXO-1 & GССTCTGCСTGGCTTTCAACTAA \\
\hline $3^{\prime}-$ LsFoxO-2 & TCCGCCAGCGACAACGGTGAT \\
\hline \multicolumn{2}{|c|}{ For RT-PCR and real-time PCR } \\
\hline Q LSFOXO-F & TCTGCCTGGCTITCAACTAA \\
\hline Q LsFoxO-R & CCGAGTCGCATCGTCTGT \\
\hline$E F-1-F$ & ССTTACCCATGTTGGATGCTTATT \\
\hline$E F-1-R$ & TGCTTCTGTCTTCCTCTITCTTCC \\
\hline$A R F-F$ & TTGGACAGTATCAAGACCCATC \\
\hline$A R F-B$ & \\
\hline
\end{tabular}

For dsRNA synthesis

dsLsFoXO-F ggatcctaatacgactcactataggGGAACGGCCTGGATGCTA

dsLSFOXO-R ggatcctaatacgactcactataggCTGCGGGTATGAAGGTGAG

dsGFP-F ggatcctaatacgactcactataggACGTAAACGGCCACAAGTTC

dsGFP-R ggatcctaatacgactcactataggTGTTCTGCTGGTAGTGGTCG 


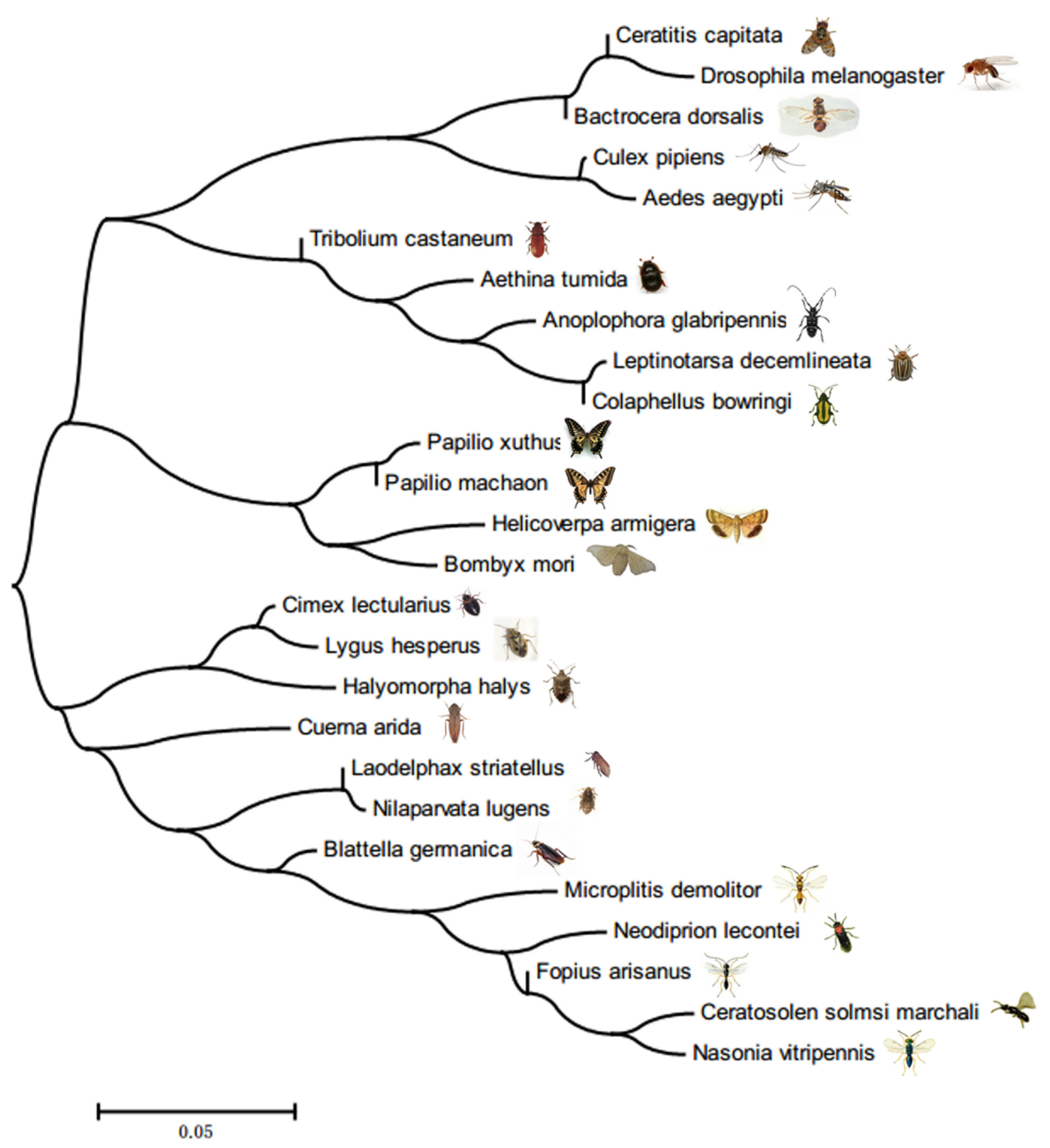

FIGURE 1 | Neighbor-Joining phylogenetic tree based on LsFoxO amino acid sequences.
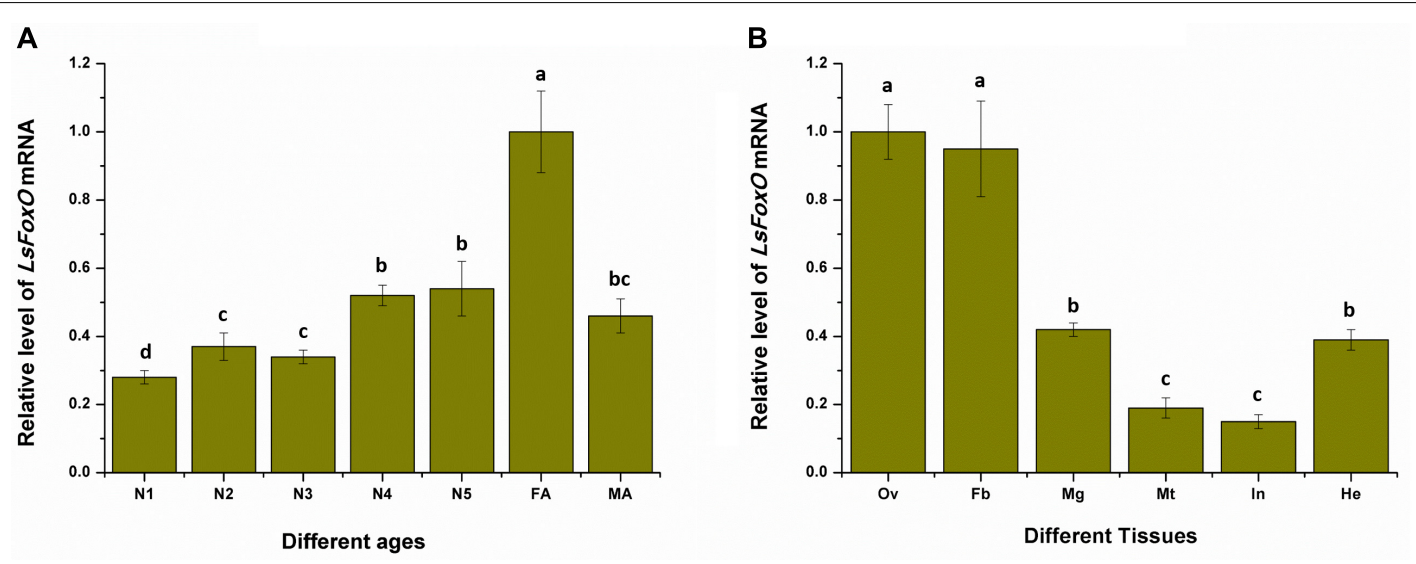

FIGURE 2 | Expression of LsFoxO gene at different developmental stages (A) and various tissues (B) were determined by qRT-PCR. (A) N1-N5, nymph of 1st to 5th; FA, second day brachypterous female adult; MA, second day brachypterous male adult. (B) Ov, ovary; Fb, fat body; Mg, midgut; Mt, malpighian tube; In, integument; He, hemolymph. Data represent mean values \pm SEM $(n=3)$, and the different letters means significant difference $(p<0.05$, Tukey's post hoc test). 
A

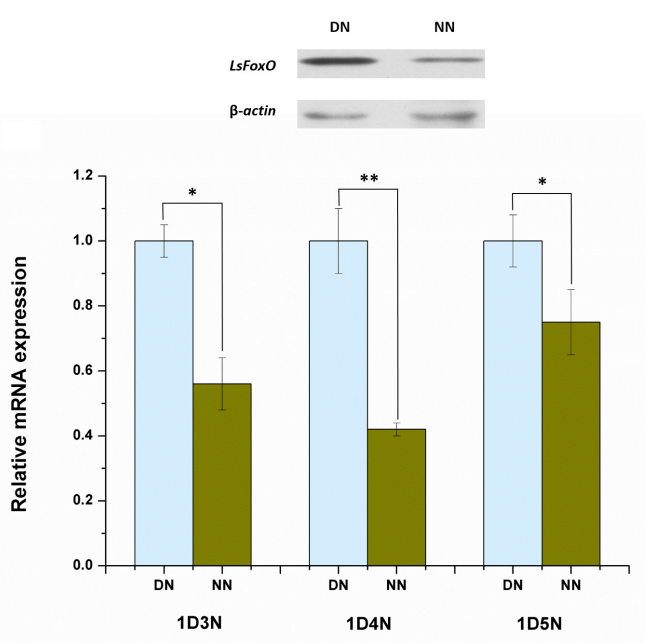

C

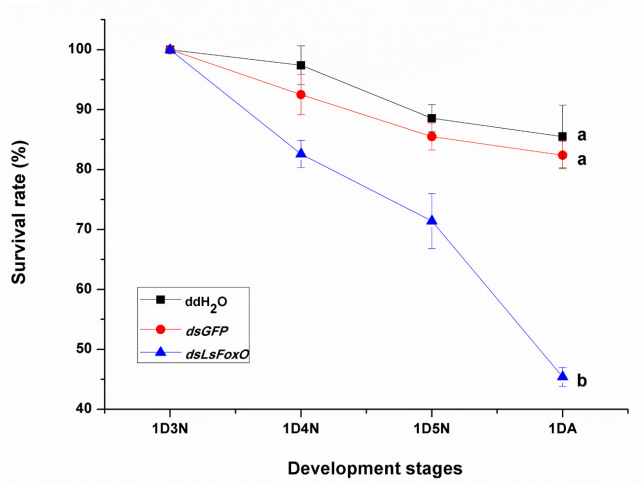

B

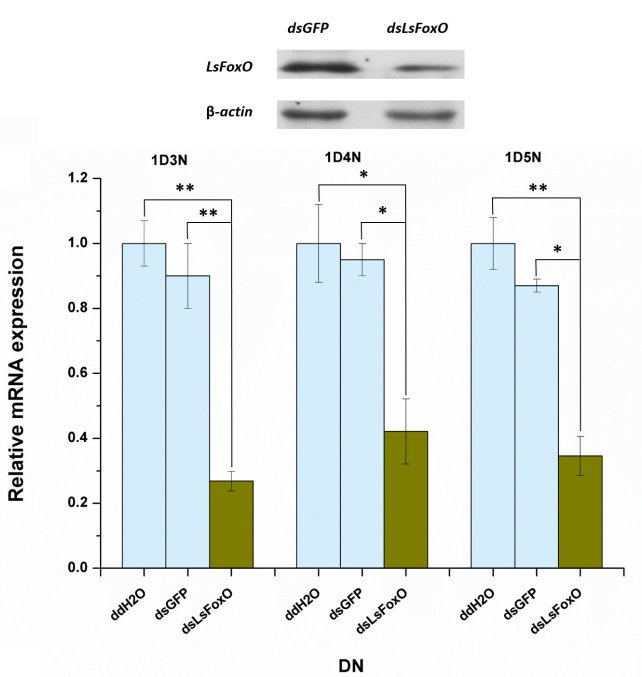

D

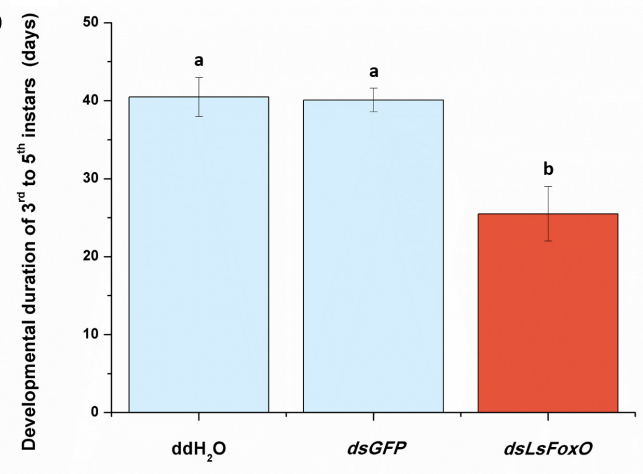

FIGURE 3 | Relative mRNA expression of LSFoxO gene were determined by qRT-PCR. (A) LsFoxO transcript levels in diapause and non-diapause nymphs, at 1st day third instar nymph (1D3N), 1st day fourth instar nymph (1D4N) and 1st day fifth instar nymph (1D5N). Inset shows Western blotting analysis of LsFoxO protein levels at 1D4N. (B) The transcript levels of $\angle s F O x O$ after RNAi in diapause nymphs. Inset shows the LsFoxO protein levels injected with dsRNA at $72 \mathrm{~h}$ postinjection after 1D4N. (C) The survival rates of diapause nymphs from 3rd instar to the initiation of adult stage. (D) The developmental duration of diapause nymphs. Three replicates were conducted, with the data presented as mean \pm SEM. Significant differences between treatment and control are indicated with asterisks ${ }^{*} p<0.05$; $\left.{ }^{* *} p<0.01\right)$, and the different letters means significant difference $(p<0.05$, Tukey's post hoc test).

\section{Quantitative Real-Time PCR Analysis}

The primers used for real-time PCR are listed in Table $\mathbf{1 .}$ The synthesized first-strand cDNA was amplified by PCR in $10 \mu \mathrm{L}$ reaction mixtures using a Light Cycler 480 system (Roche, United States), and ADP-ribosylation factor $(A R F)$ and elongation factor-1 (EF-1) genes were used as an internal standard (Wan et al., 2014). The quantitative variation was calculated using three independent biological samples by a relative quantitative method $\left(2^{-\Delta \Delta \mathrm{CT}}\right)$.

\section{Western Blotting}

The proteins were separated using 12\% SDS-PAGE gel and transferred to PVDF membranes $(0.4 \mu \mathrm{m}$; EMD Millipore, Hayward, CA, United States), and the membranes were immunoblotted with anti-LsFoxO serum (1:3000) prepared by our laboratory. IgG goat anti-mouse and goat anti-rabbit antibodies conjugated with HRP were used for secondary antibodies (1:5000, Abcam, Cambridge,
United Kingdom), and the membranes were visualized by ECL.

\section{RNA Interference and Sampling}

The dsRNA of LsFoxO was produced using the T7 RiboMAXTM Express RNAi System (Promega, Sunnyvale, CA, United States). After synthesis, dsLsFoxO (MF197906, $431 \mathrm{bp)}$ and $d s G F P$ (DQ389577, 495 bp) were quantified by an ultramicrospectrophotometers (NanoDrop 2000, Thermo Fisher, Scotts Valley, CA, United States) and were maintained at $-80^{\circ} \mathrm{C}$ until use (Table 1). The sequence was verified by sequencing (Sangon Biotech, Shanghai, China). Before injection, the dsRNA and phenol red solution were mixed for observations. Under carbon dioxide anesthesia, nymphs were immobilized on the agarose injection plate with the ventral side upward, under $\mathrm{CO}_{2}$ anesthesia. The purified $d s L s F o x O$ and $d s G F P$ were slowly injected on one side of the metathorax using the Nanoject II (Drummond, Broomall, PA, United States). The injected individuals were 

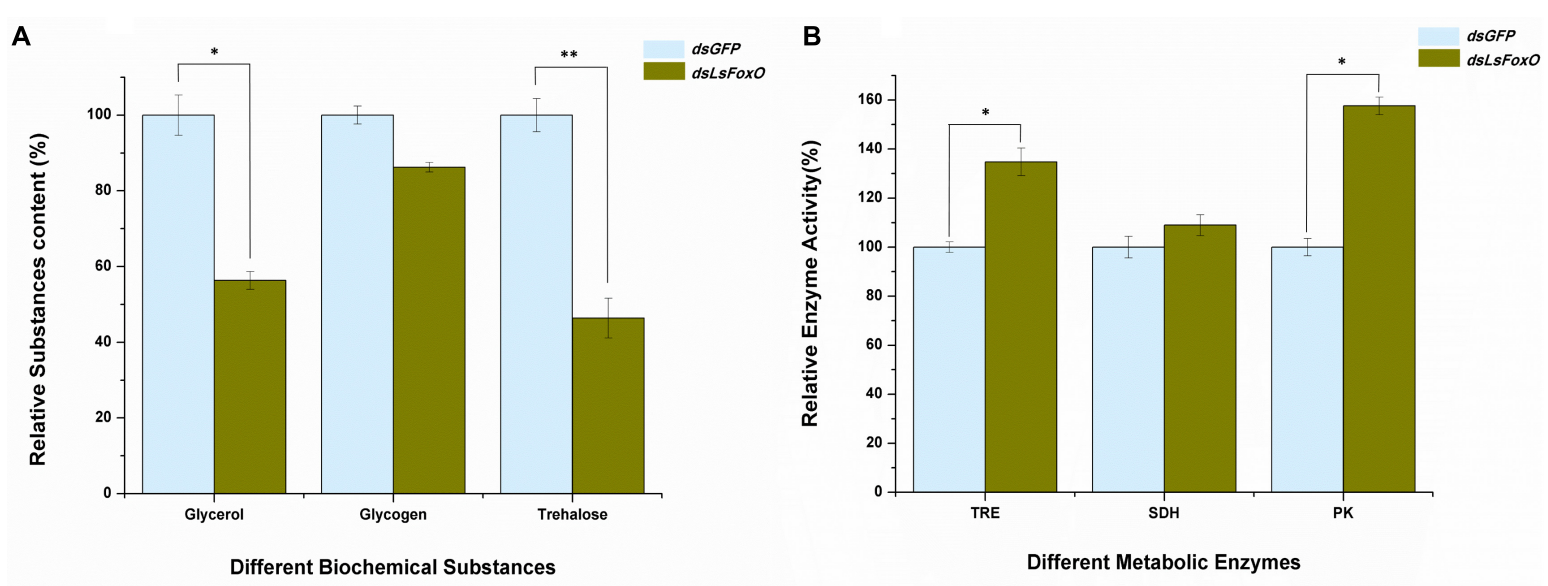

C

D

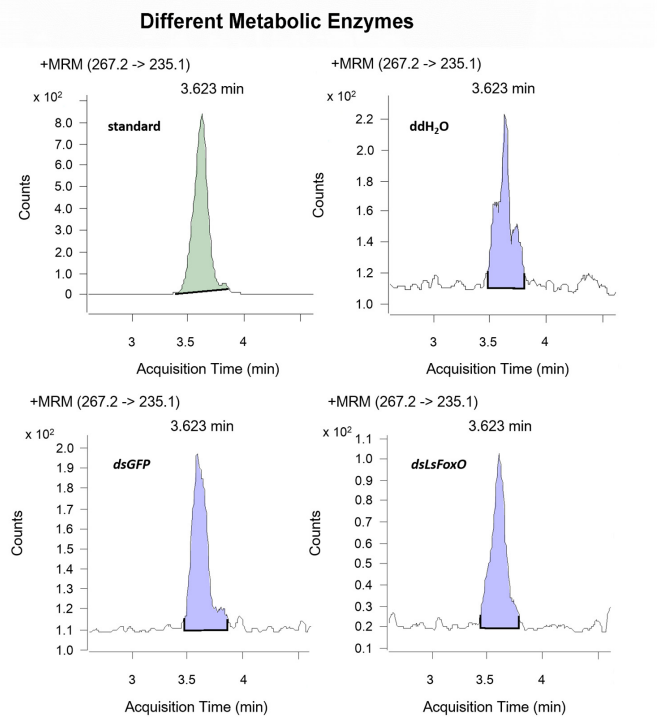

FIGURE 4 | Relative physiological and biochemical changes. (A) Relative substance content, (B) Relative enzyme activity, and (C) the JH III titer were analyzed at $72 \mathrm{~h}$ post-injection by different treatment at 1D4N. (D) The representative chromatograms of JH III. Three replicates were conducted, with the data presented as mean \pm SEM. Significant differences between treatment and control are indicated with asterisks $\left({ }^{*} p<0.05 ;{ }^{* *} p<0.01\right)$, and the different letters means significant difference ( $p<0.05$, Tukey's post hoc test).

placed in a glass tube (length: $200 \mathrm{~mm}$; diameter: $25 \mathrm{~mm}$ ) on fresh rice seedlings for further observation. Data on developmental duration were recorded every day until the adult emerged.

\section{Assessment of Metabolic Enzyme Activities and Biochemical Substances}

To clarify the physiological adaptation of first day fourthinstar diapause nymphs with $d s L s F o x O$ or $d s G F P$ treated. Glycogen (MAK016) and triglyceride (TR0100) were measured by commercial kits (Sigma-Aldrich Co., LLC., United States), and trehalose (K-TREH) was measured by commercial kits (Megazyme, Ireland). Some cold tolerance-related metabolic enzymes, such as TRE, SDH and PK were quantified. The activities of metabolic enzymes were measured by commercial kits (Suzhou Comin Biotechnology Co., Ltd., Suzhou, China), and the absorbance of TRE, SDH and PK were measured at $340 \mathrm{~nm}$.

\section{Quantitative Determination of Hormone}

Laodelphax striatellus samples were separately ground in grinder and ultra sonicated with methanol and isooctane. After centrifugation at $12,000 \mathrm{~g}$ for $10 \mathrm{~min}$, the upper layer was transferred into a test tube, the ultrasound-assisted extraction was repeated twice. The combined extracts were evaporated to dryness in a $40^{\circ} \mathrm{C}$ water-bath under a stream of nitrogen. The residue was reconstituted in methanol, then transferred to injection vials and analyzed using HPLC-MS/MS, (Agilent 6420; Waldbronn, Germany). JH III was separated using gradient elution and the hormone titer was expressed as ng per mg body weight.

\section{Transcriptomic Analyses}

The total RNA was extracted using the E.Z.N.A. ${ }^{\circledR}$ Total RNA Kit II (Omega Bio-Tek, Norcross, GA, United States) according to the manufacturer's instructions. To obtain ideal gene expression information after the RNAi, first day fourth-instar diapause 

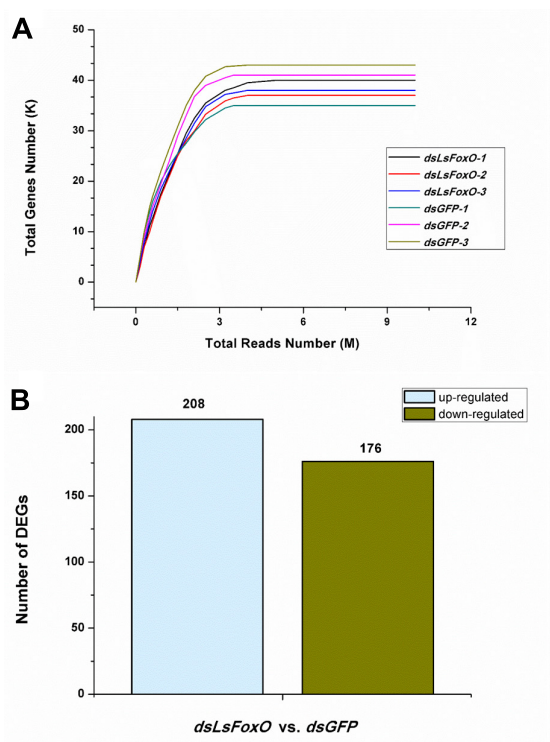

C

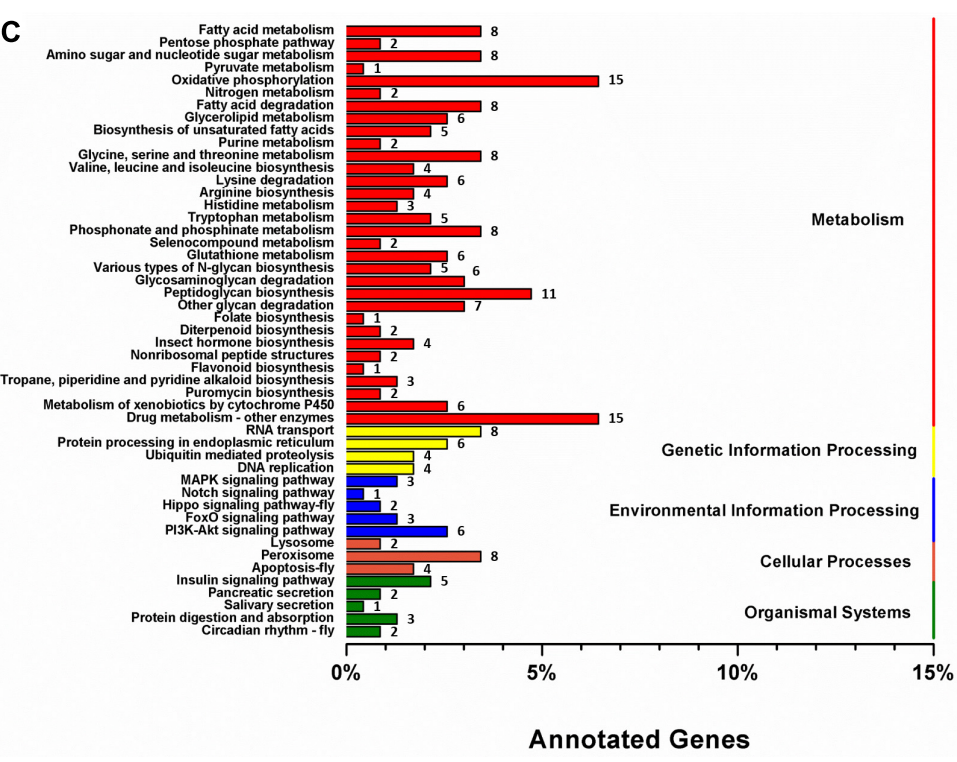

FIGURE 5 | Transcriptomic analysis of the DEGs data in LsFoxO knockdown diapause nymphs. (A) Simulated diagram of saturation test of sequencing data. X axis indicates the number of reads $\left(10^{6}\right), Y$ axis indicates the number of detected genes $\left(10^{3}\right)$ and FPKM $\geq 0.1$. (B) Number of significantly changed annotated DEGs, the conditions for genes was FDR $\leq 0.01$ and FC $\geq 2$. (C) The distribution of pathways of DEGs annotated in the KEGG data library.

nymphs were injected with $d s L s F o x O$ or $d s G F P$, and after $48 \mathrm{~h}$, the samples were used for transcriptomic analyses. The genes differentially expressed between the two samples were identified using an algorithm as previously described (Schulze et al., 2012). Each cDNA library was sequenced using the Illumina sequencing platform (Hiseq 2500; Illumina, Hayward, CA, United States) according to the manufacturer's instructions.

\section{Statistical Analyses}

The statistical analyses were performed using SPSS 17.0 software, differences between treatments levels were examined using ANOVA, followed by Tukey's analysis.

\section{RESULTS}

\section{Isolation and Characterization of $L s F o x O$ cDNA}

The full-length LsFoxO sequence was 2,766 bp (GenBank accession No: MF197906) and had an open reading frame (ORF) of 1,275 bp, which encoded a protein of 424 amino acids with a predicted mass of approximately $46.41 \mathrm{kDa}$ and an isoelectric point of 7.04, with a $5^{\prime}$-untranslated region (UTR) of $781 \mathrm{bp}$ and a $3^{\prime}$-UTR of 710 bp (Supplementary Figure 1).

A phylogenetic tree was constructed based on the fulllength sequences of known FoxO genes from insects and other organisms (Figure 1). The BLAST results showed that the amino acid sequence of LsFoxO had the greatest similarity to FoxO from Nilaparvata lugens (Hemiptera) (88\%) (XP_022196038), Halyomorpha halys (Hemiptera) (69\%) (XP_014290452), Cimex lectularius (Hemiptera) (69\%) (XP_014254467), Helicoverpa armigera (Lepidoptera) (58\%) (XP_021186671), Pieris rapae (Lepidoptera) (56\%) (XP_022130764).

\section{The Spatiotemporal Expression of LsFoxO}

To determine whether LsFoxO was present during developmental stages and in various tissues in the female adult L. striatellus, total RNA from each sample was isolated. We used qRT-PCR to characterize the LsFoxO gene's expression pattern in all of the developmental stages. The LsFoxO mRNA expression level was high in the 4 th-5th instar nymphal period, but the expression level was higher in the female adult period (Figure 2A). The expression of LsFoxO mRNA was investigated in various tissues in adult females. LsFoxO was highly expressed in the fat body, hemolymph and ovary, with lower expression levels in the integument, Malpighian tube and midgut (Figure 2B).

\section{Effect of Knocking Down LsFoxO on Nymphal Performance}

To evaluate the effects of an LsFoxO knockdown on nymphal diapause, we used a microinjection-based RNA interference (RNAi) method. Before analyzing the RNAi efficiency, we detected the LsFoxO mRNA expression level in diapausal nymphs (DNs) and non-diapausal nymphs (NNs), including 1st day 3rd instar nymph (1D3N), 1st day 4th instar nymph (1D4N) and 1st day 5th instar nymph (1D5N). The LsFoxO mRNA expression levels were significantly up-regulated in DNs, and the protein levels were also increased in DNs at $1 \mathrm{D} 4 \mathrm{~N}$ (Figure 3A). At $48 \mathrm{~h}$ after an injection of $d s L s F o x O$, the expression of LsFoxO decreased by 73.19, 57.90, and $65.40 \%$ in the three different periods, respectively, compared with 


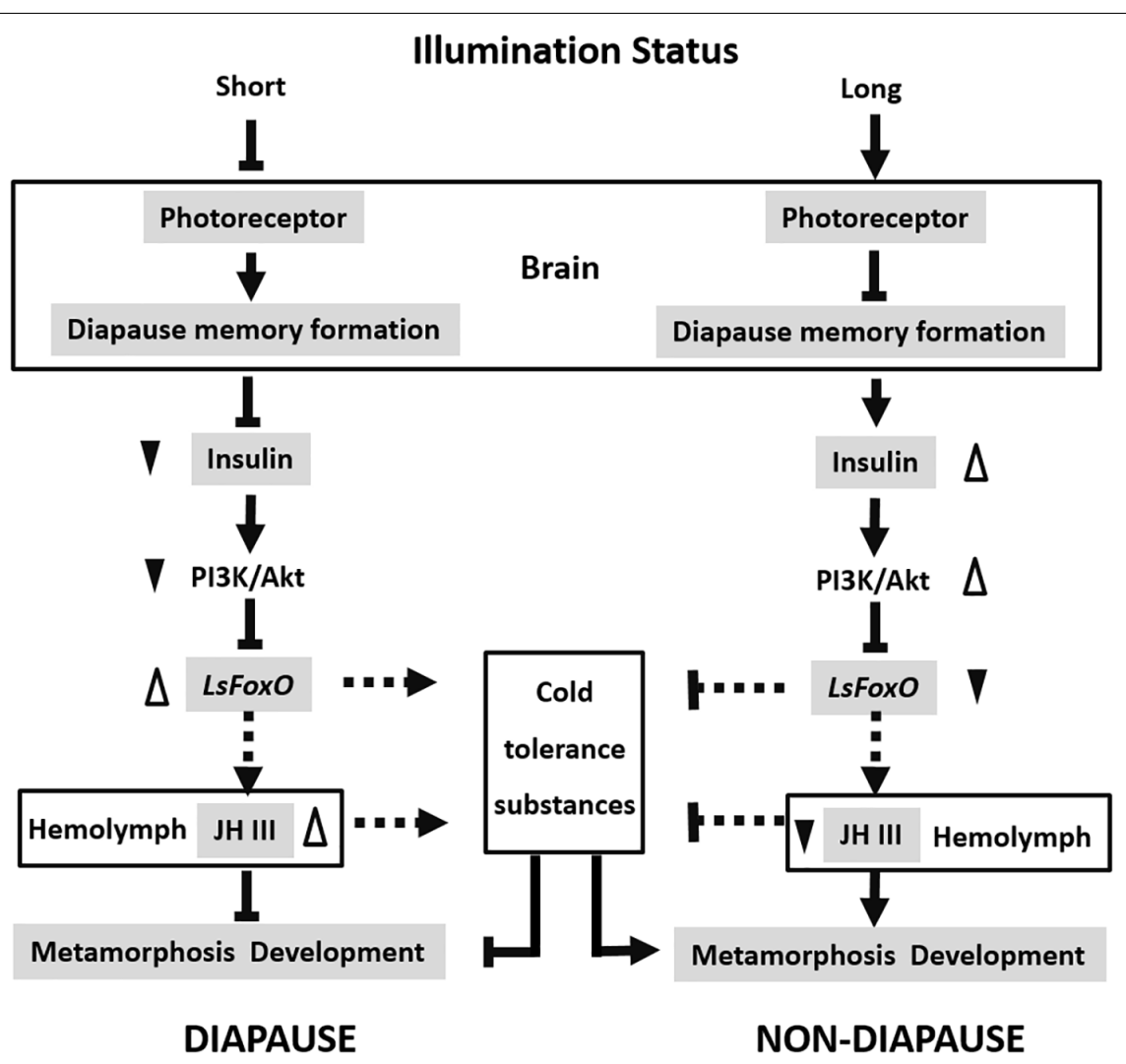

FIGURE 6 | Proposed model of the different photoperiodic signals interacts with LsFoxO to regulate the nymphal diapause. Short illumination status decrease photoreceptor activity, and the photoperiodic information will subsequently be stored by a special memory formation process in the brain. The insulin signaling pathway is repressed, which stimulate the LsFoxO activity. LsFoxO up-regulate some cold tolerance substances accumulation and JH III titers in the hemolymph. Long illumination status results in opposite effects. The model provides an explanation for different photoperiodic signals interacts with $L s F o x O$ to regulate the nymphal diapause.

an injection of $\mathrm{ddH}_{2} \mathrm{O}$. Western blot analyses showed that the LsFoxO protein levels also decreased after injections of $d s L s F o x O$ (Figure 3B). These results indicated that the RNAi was effective.

The injection of $d s L s F o x O$ significantly decreased the nymphal survival rate to $45.4 \%$, and the nymphal period from 3 rd to 5 th instar was significantly shortened after injection. By contrast, over $82 \%$ of the nymphs injected with water or $d s G F P$ survived (Figure 3C). The average duration to adult eclosion of nymphs injected with dsLsFoxO was 25.5 days, which was significantly shorter than the mean periods of other treatments at $20^{\circ} \mathrm{C}$ under short day-length conditions (10 L:14 D) (Figure 3D).

\section{Assessment of Physiological and Biochemical Changes}

Diapause regulates several physiological and biochemical mechanisms, particularly modifying the activities of some cold-tolerance substances and metabolic enzymes. At $72 \mathrm{~h}$ after an injection of $d s L s F o x O$, the glycerol and trehalose levels significantly decreased (43.63 and 53.62\%, respectively) (Figure 4A), the enzymatic activities of TRE and PK significantly increased (34.79 and 57.62\%, respectively) (Figure 4B), and the juvenile hormone (JH) III titer indicated that the hormone levels were significantly decreased (Figures 4C,D).

\section{Global Changes at the Transcript Level After LsFoxO RNAi}

A total of $38,547,651$ clean pair-end reads were generated by Illumina sequencing and were de novo assembled into 42,273 unigenes, with an $\mathrm{N}_{50}$ length of 1,357 bp (Supplementary Table 1). The saturated gene number achieved with the increase in sequenced reads indicates that sufficient and effective information was applied in this study (Figure 5A). L. striatellus lacks a reference genome; therefore, 31,254 unigenes were annotated from the Clusters of Orthologous Groups (COG, 10,671), GO (10,083), Kyoto Encyclopedia of Genes and Genomes (KEGG, 9,794), EuKaryotic Orthologous Groups (KOG, 18,483), Protein family (Pfam, 15,526), SwissProt $(9,384)$ and NR $(27,325)$ databases using a cut-off $e$-value of $10^{-5}$ (Supplementary Table 2). The differentially expressed genes (DEGs) following RNAi treatment were analyzed according to the gene expression level (FPKM). Based on the DEG analysis, 384 genes had significantly different expression levels between the $d s L s F o x O$ - and $d s G F P$-treated libraries, including 208 up- and 
176 down-regulated genes (Figure 5B). According to the KEGG analysis, most of the DEGs correlated with metabolic and environmental information processes, including carbohydrate metabolism, amino acid metabolism, fatty acid metabolism and the PI3K-Akt signaling pathway (Figure 5C).

\section{DISCUSSION}

Diapause usually occurs during a specific stage, such as embryo, larvae/nymph, pupae or adult stages (Jiang et al., 2010; Liu et al., 2010; Kobayashi et al., 2014). Some research have found that diapause occurs in several nymphal stages of $L$. striatellus, and which varies with geographic location (Wang et al., 2014; Hou et al., 2016). In our previous study, we discovered that the overwintering diapause occurred in 3rd to 5th instars, with the 4th instar nymph being the predominant diapause stage (Zhai et al., 2018). Diapause is a complex physiological response process with many regulatory features, number of genes, proteins, and metabolites that are differentially expressed in diapause (Zhang et al., 2013). Diapause is regulated by the $\mathrm{JH}$, and ecdysone in diverse species. A high $\mathrm{JH}$ titer inhibits ecdysone secretion during diapause maintenance, and the $\mathrm{JH}$ titer decreases significantly during late diapause. Hormone signaling regulates nymphal diapause in L. striatellus (Zhai et al., 2017). In $D$. melanogaster, $\mathrm{JH}$ and ecdysone synthesis are regulated by the insulin signaling pathway (Colombani et al., 2005; Tu et al., 2005), and insulin signaling is a regulator of diapause (Sim and Denlinger, 2013). FoxO is a well-known regulator of life span extension (Martins et al., 2016), insulin activates p-Akt levels repress FoxO activity, which activate other cross-talk genes that promote the insects' development. In contrast, a low level of insulin signaling represses PI3K/Akt and increases the FoxO activity, which regulates life-span extension and generates the diapause phenotype (Sim et al., 2015). High levels of p-Akt fail to phosphorylate FoxO through PRMT1-mediated methylation, blocking FoxO phosphorylation reduces FoxO protein degradation, thus promoting the accumulation of FoxO in brains, which leads to pupal diapause in Helicoverpa armigera (Zhang et al., 2017). The overexpression of FoxO during early larval stages inhibits development and extends life-spans. Our results, in which LsFoxO mRNA expression and protein levels were significantly up-regulated in diapause nymphs, corroborates previous findings (Figure 3A).

After evaluating the spatiotemporal expression of the LsFoxO gene in L. striatellus using qRT-PCR, we found that higher expression levels were detected in 4th-5th instar nymphs and the female adults. FoxO is expressed during all of the developmental stages in other insects (Hwangbo et al., 2004; Carter and Brunet, 2007). In the present study, the injection of dsLsFoxO significantly inhibited the gene's mRNA in the three different nymphal periods and the protein level in the 4th instar nymph (Figure 3B). Compared with these results, the developmental duration of 3rd to 5th instar nymphs may be a better indicator of the functional relationship between LsFoxO and nymphal diapause. Diapause is a form of dormancy used by insects to survive under adverse environmental conditions and is usually related to some cold-tolerance substances, such as glycerol, trehalose and glucose, which can improve the insects' cold tolerance and help overcome severe winter environments (Denlinger, 2002; Zhai et al., 2016). TRE is a key enzyme in trehalose hydrolysis and changes in the activity of this enzyme directly affect energy metabolism (Kamei et al., 2011). PK is the key enzymes in the glycolytic pathway, and PK mediates the conversion of phosphoenolpyruvic acid and ADP into pyruvic acid and ATP (Rider et al., 2011). Here, TRE and PK levels significantly increased after an injection of $d s L s F o x O$, and the glycerol and trehalose levels significantly decreased (Figures 4A,B). Thus, the diapausal nymphs had increased their cold tolerance through the accumulation of cold-tolerance substances.

Targeting a gene using RNAi may reveal its function, and this method has been most recently used for agricultural pest control (Baum et al., 2007; Mao et al., 2007). However, there have been limited studies on the global changes in the mRNA profile that occur after RNAi targeting of a specific gene (Wang et al., 2008). We determined previously that 4th instar nymphs represent the main diapausal. Therefore, we selected 1D4N diapausal nymphs to inject with dsRNA and for transcriptomic analyses. We showed that in 384 annotated DEGs between LsFoxO-RNAi and control samples, 208 and 176 genes were up- and downregulated, respectively (Figure 5B). Among the down-regulated genes, we found the RNAi target gene LsFoxO (c11725.graph_c0), indicating that the RNAi was effective. To review the global changes in the signaling pathway after RNAi, we used the KEGG analytical method. Most of the DEGs correlated with metabolic and environmental information processes, including carbohydrate metabolism, amino acid metabolism, fatty acid metabolism and the PI3K-Akt signaling pathway (Figure 5C). The latter is a signal transduction pathway that promotes survival and growth in response to extracellular signals and is a key component of the insulin signaling pathway. Insulin activates PI3K-Akt, and high p-Akt levels repress FoxO activity and activate other genes that inhibit diapause.

In summary, FoxO is a key downstream regulator that acts as a key developmental switch in insect diapause, which is regulated by the insulin signaling pathway. LsFoxO can regulate some cold-tolerance substances and JH III titers in the hemolymph to control the nymphal diapause status. This supports the conclusion that physiological levels of FoxO are beneficial for diapause. We propose a model to explain how different photoperiod signals interact with $L s F o x O$ to regulate nymphal diapause in L. striatellus (Figure 6). There are still many issues to be studied in the future, such as how LsFoxO regulates $\mathrm{JH}$ expression and the accumulation of some cold-tolerance substances? The present results offer new insights into nymphal diapause and contribute to a comprehensive understanding of insect diapause.

\section{AUTHOR CONTRIBUTIONS}

Z-JY, X-LD, YY, and Y-FZ conceived and designed the experiments. Z-JY, KK, G-AW, HC, and XY-D preformed the 
experiments. Z-JY, LZ, and Y-FZ analyzed the data and wrote the manuscript. All authors read and approved the final manuscript.

\section{FUNDING}

This work was financially supported through a grant from the National Natural Science Foundation of China (31401803) and

\section{REFERENCES}

Anspaugh, D. D., and Roe, R. M. (2005). Regulation of juvenile hormone epoxide hydrolase versus juvenile hormone esterase activity in the cabbage looper, Trichoplusia ni, by juvenile hormone and xenobiotics. J. Insect Physiol. 51, 523-535. doi: 10.1016/j.jinsphys.2004.12.008

Baum, J. A., Bogaert, T., Clinton, W., Heck, G. R., Feldmann, P., Ilagan, O., et al. (2007). Control of coleopteran insect pests through RNA interference. Nat. Biotechnol. 25, 1322-1326. doi: 10.1038/nbt1359

Carter, M. E., and Brunet, A. (2007). FOXO transcription factors. Curr. Biol. 17, R113-R114. doi: 10.1016/j.cub.2007.01.008

Colombani, J., Bianchini, L., Layalle, S., Pondeville, E., Dauphin-Villemant, C., Antoniewski, C., et al. (2005). Antagonistic actions of ecdysone and insulins determine final size in Drosophila. Science 310, 667-670. doi: 10.1126/science. 1119432

Denlinger, D. L. (2002). Regulation of diapause. Annu. Rev. Entomol. 47, 93-122. doi: 10.1146/annurev.ento.47.091201.145137

Denlinger, D. L. (2008). Why study diapause? Entomol. Res. 38, 1-9. doi: 10.1111/j. 1748-5967.2008.00139.x

Eizaguirre, M., Prats, J., Abellana, M., Carmen López, Llovera, M., and Canela, R. (1998). Juvenile hormone and diapause in the Mediterranean corn borer, Sesamia nonagrioides. J. Insect Physiol. 44, 419-425. doi: 10.1016/S00221910(98)00019-5

Fan, W. T., Zhong, Y. S., Qin, M. Y., Lin, B. M., Chen, F. Y., Yan, H. C., et al. (2017). Differentially expressed microRNAs in diapausing versus $\mathrm{HCl}$ treated Bombyx embryos. PLoS One 12:e0180085. doi: 10.1371/journal.pone. 0180085

Hahn, D. A., and Denlinger, D. L. (2011). Energetics of insect diapause. Annu. Rev. Entomol. 56, 103-121. doi: 10.1146/annurev-ento-112408-085436

Hou, Y. Y., Xu, L. Z., Wu, Y., Wang, P., Shi, J. J., and Zhai, B. P. (2016). Geographic variation of diapause and sensitive stages of photoperiodic response in Laodelphax striatellus Fallén (Hemiptera: Delphacidae). J. Insect Sci. 16, 1-7. doi: 10.1093/jisesa/iev161

Hwangbo, D. S., Gersham, B., Tu, M. P., Palmer, M., and Tatar, M. (2004). Drosophila dFOXO controls lifespan and regulates insulin signalling in brain and fat body. Nature 429, 562-566. doi: 10.1038/nature02549

Jiang, X. F., Huang, S. H., Luo, L. Z., Liu, Y., and Zhang, L. (2010). Diapause termination, post-diapause development and reproduction in the beet webworm, Loxostege sticticalis (Lepidoptera: Pyralidae). J. Insect Physiol. 56, 1325-1331. doi: 10.1016/j.jinsphys.2010.04.016

Jindra, M., Palli, S. R., and Riddiford, L. M. (2013). The juvenile hormone signaling pathway in insect development. Annu. Rev. Entomol. 58, 181-204. doi: 10.1146/ annurev-ento-120811-153700

Junger, M. A., Rintelen, F., Stocker, H., Wasserman, J. D., Vegh, M., Radimerski, T., et al. (2003). The Drosophila forkhead transcription factor FOXO mediates the reduction in cell number associated with reduced insulin signaling. J. Biol. 2:20. doi: 10.1186/1475-4924-2-20

Kamei, Y., Hasegawa, Y., Niimi, T., Yamashita, O., and Yaginuma, T. (2011). Trehalase-2 protein contributes to trehalase activity enhanced by diapause hormone in developing ovaries of the silkworm, Bombyx mori. J. Insect Physiol. 57, 608-613. doi: 10.1016/j.jinsphys.2010.10.001

Kauranen, H., Ala-Honkola, O., Kankare, M., and Hoikkala, A. (2016). Circadian clock of Drosophila montana is adapted to high variation in summer day lengths and temperatures prevailing at high latitudes. J. Insect Physiol. 89, 9-18. doi: 10.1016/j.jinsphys.2016.03.005 the Shandong Provincial Natural Science Foundation, China (ZR2014CQ014).

\section{SUPPLEMENTARY MATERIAL}

The Supplementary Material for this article can be found online at: https://www.frontiersin.org/articles/10.3389/fphys. 2018.01654/full\#supplementary-material

Kisimoto, R. (1989). Flexible diapause response to photoperiod of a laboratory selected line in the small brown planthopper, Laodelphax Striatellus Fallén. Appl. Entomol. Zool. 24, 157-159. doi: 10.1303/aez.24.157

Kobayashi, N., Takahashi, M., Kihara, S., Niimi, T., Yamashita, O., and Yaginuma, T. (2014). Cloning of cDNA encoding a Bombyx mori homolog of human oxidation resistance 1 (OXR1) protein from diapause eggs, and analyses of its expression and function. J. Insect Physiol. 68, 58-68. doi: 10.1016/ j.jinsphys.2014.06.020

Kostal, V. (2006). Eco-physiological phases of insect diapause. J. Insect Physiol. 52, 113-127. doi: 10.1016/j.jinsphys.2005.09.008

Li, H. Y., Wang, T., Yang, Y. P., Geng, S. L., and Xu, W. H. (2017). TGF- $\beta$ signaling regulates $\mathrm{p}$-Akt levels via PP2A during diapause entry in the cotton bollworm, Helicoverpa armigera. Insect Biochem. Mol. Biol. 87, 165-173. doi: 10.1016/j.ibmb.2017.07.003

Liu, Z. D., Gong, P. Y., Li, D. M., and Wei, W. (2010). Pupal diapause of Helicoverpa armigera (Hubner) (Lepidoptera Noctuidae) mediated by larval host plants pupal weight is important. J. Insect Physiol. 56, 1863-1870. doi: 10.1016/j. jinsphys.2010.08.007

Mao, Y. B., Cai, W. J., Wang, J. W., Hong, G. J., Tao, X. Y., Wang, L. J., et al. (2007). Silencing a cotton bollworm P450 monooxygenase gene by plant-mediated RNAi impairs larval tolerance of gossypol. Nat. Biotechnol. 25, 1307-1313. doi: $10.1038 /$ nbt1352

Martins, R., Lithgow, G. J., and Link, W. (2016). Long live FOXO: unraveling the role of FOXO proteins in aging and longevity. Aging Cell 15, 196-207. doi: 10.1111 /acel.12427

Matsunaga, Y., Matsukawa, T., Iwasaki, T., Nagata, K., and Kawano, T. (2018). Comparison of physiological functions of antagonistic insulin-like peptides, INS-23 and INS-18, in Caenorhabditis elegans. Biosci. Biotechnol. Biochem. 82, 90-96. doi: 10.1080/09168451.2017.1415749

Otuka, A. (2013). Migration of rice planthoppers and their vectored emerging and novel rice viruses in East Asia. Front. Microbiol. 4:309. doi: 10.3389/fmicb.2013. 00309

Rider, M. H., Hussain, N., Dilworth, S. M., Storey, J. M., and Storey, K. B. (2011). AMP-activated protein kinase and metabolic regulation in coldhardy insects. J. Insect Physiol. 57, 1453-1462. doi: 10.1016/j.jinsphys.2011.07.006

Rozsypal, J., Koštál, V., Zahradníčková, H., and Šimek, P. (2013). Overwintering strategy and mechanisms of cold tolerance in the codling moth (Cydia pomonella). PLoS One 8:e61745. doi: 10.1371/journal.pone.0061745

Schulze, S. K., Kanwar, R., Gölzenleuchter, M., Therneau, T. M., and Beutler, A. S. (2012). SERE: single-parameter quality control and sample comparison for RNA-Seq. BMC Genomics 13:524. doi: 10.1186/1471-2164-13-524

Sim, C., and Denlinger, D. L. (2008). Insulin signaling and FOXO regulate the overwintering diapause of the mosquito Culex pipiens. Proc. Natl. Acad. Sci. U.S.A. 105, 6777-6781. doi: 10.1073/pnas.0802067105

Sim, C., and Denlinger, D. L. (2013). Insulin signaling and the regulation of insect diapause. Front. Physiol. 4:189. doi: 10.3389/fphys.2013.00189

Sim, C., Kang, D. S., Kim, S., Bai, X., and Denlinger, D. L. (2015). Identification of FOXO targets that generate diverse features of the diapause phenotype in the mosquito Culex pipiens. Proc. Natl. Acad. Sci. U.S.A. 112, 3811-3816. doi: 10.1073/pnas.1502751112

Tu, M. P., Yin, C. M., and Tatar, M. (2005). Mutations in insulin signaling pathway alter juvenile hormone synthesis in Drosophila melanogaster. Gen. Comp. Endocrinol. 142, 347-356. doi: 10.1016/j.ygcen.2005.02.009

Wan, P. J., Jia, S., Li, N., Fan, J. M., and Li, G. Q. (2014). RNA interference depletion of the Halloween gene disembodied implies its potential application 
for management of planthopper Sogatella furcifera and Laodelphax striatellus. PLoS One 9:e86675. doi: 10.1371/journal.pone.0086675

Wang, L., Lin, K., Chen, C., Fu, S., and Xue, F. S. (2014). Diapause induction and termination in the small brown planthopper, Laodelphax striatellus (Hemiptera: Delphacidae). PLoS One 9:e107030. doi: 10.1371/journal.pone. 0107030

Wang, S. W., Stevenson, A. L., Kearsey, S. E., Watt, S., and Bähler, J. (2008). Global role for polyadenylation-assisted nuclear RNA degradation in posttranscriptional gene silencing. Mol. Cell Biol. 28, 656-665. doi: 10.1128/ MCB.01531-07

Williams, K. D., Busto, M., Suster, M. L., So, A. K., Ben-Shahar, Y., Leevers, S. J., et al. (2006). Natural variation in Drosophila melanogaster diapause due to the insulin-regulated PI3-kinase. Proc. Natl. Acad. Sci. U.S.A. 103, 15911-15915. doi: $10.1073 /$ pnas. 0604592103

Xu, W. H., Lu, Y. X., and Denlinger, D. L. (2012). Cross-talk between the fat body and brain regulates insect developmental arrest. Proc. Natl. Acad. Sci. U.S.A. 109, 14687-14692. doi: 10.1073/pnas.1212 879109

Zhai, Y. F., Lin, Q. C., Zhang, J. P., Zhang, F., Zheng, L., and Yu, Y. (2016). Adult reproductive diapause in Drosophila suzukii females. J. Pest Sci. 89, 679-688. doi: $10.1007 / s 10340-016-0760-9$

Zhai, Y. F., Zhang, X. Y., Yin, Z. J., Zhu, Q. S., Tao, M., Yu, Y., et al. (2018). Nymphal diapause in Laodelphax striatellus (Hemiptera: Delphacidae). J. Entomol. Sci. 53, 107-122. doi: 10.18474/JES17-140.1
Zhai, Y. F., Zhang, Z. M., Gao, H. H., Chen, H., Sun, M., Zhang, W. Q. et al. (2017). Hormone signaling regulates nymphal diapause in Laodelphax striatellus (Hemiptera: Delphacidae). Sci. Rep. 7:13370. doi: 10.1038/s41598017-13879-y

Zhang, Q., Lu, Y. X., and Xu, W. H. (2013). Proteomic and metabolomic profiles of larval hemolymph associated with diapause in the cotton bollworm, Helicoverpa armigera. BMC Genomics 14:751. doi: 10.1186/1471-216414-751

Zhang, X. S., Wang, T., Lin, X. W., Denlinger, D. L., and Xu, W. H. (2017). Reactive oxygen species extend insect life span using components of the insulinsignaling pathway. Proc. Natl. Acad. Sci. U.S.A. 114, 7832-7840. doi: 10.1073/ pnas. 1711042114

Conflict of Interest Statement: The authors declare that the research was conducted in the absence of any commercial or financial relationships that could be construed as a potential conflict of interest.

Copyright () 2018 Yin, Dong, Kang, Chen, Dai, Wu, Zheng, Yu and Zhai. This is an open-access article distributed under the terms of the Creative Commons Attribution License (CC BY). The use, distribution or reproduction in other forums is permitted, provided the original author(s) and the copyright owner(s) are credited and that the original publication in this journal is cited, in accordance with accepted academic practice. No use, distribution or reproduction is permitted which does not comply with these terms. 